\title{
TRAMPOline: a Temporal Relative Abundance-focused multi-sPecies Occupancy model, illustrated using fossils
}

\author{
Trond Reitan ${ }^{1}$, Torbjørn Ergon ${ }^{1}$, and Lee Hsiang Liow ${ }^{1}$ \\ ${ }^{1} \mathrm{UiO}$
}

February 22, 2021

\begin{abstract}
The occupancy and relative abundance of species are temporally varying. Estimating these, given incomplete and biased sampling is challenging, not least for fossilized organisms, where preservation is an additional issue. Here, we describe a relative abundance-focused multi-species occupancy model (TRAMPOline) in a hierarchical Bayesian framework. We designed our model on the basis of the need to understand the dynamics of several focal species over 2.3 million years, by drawing on additional information provided by non-focal species observed in the same fossilized community. We expanded our model by adding random effects of species and time intervals (geological formations) and explored potential explanatory factors (paleoenvironmental proxies) and temporal autocorrelation that could provide extra information on unsampled geological time intervals. Our new model, set in an occupancy modeling framework widely used in ecology but little applied in paleoecology, is applicable across a wide range of questions on species-level dynamics in contemporary and palaeoecological community settings.
\end{abstract}

\section{Hosted file}

Reitan_Ergon_Liow_occupancy_ms_revised.pdf available at https://authorea.com/users/ 397278/articles/510209-trampoline-a-temporal-relative-abundance-focused-multi-speciesoccupancy-model-illustrated-using-fossils 